\title{
Inflammatory myofibroblastic tumor of lung with tuberculosis
}

\begin{abstract}
Inflammatory myofibroblastic tumor (IMT) of lung is a rare benign malignancy of lung. It histology is varied, there is combination of stromal, myoepithelial and fibrous histiocytes and plasma cells on microscopy. In this case report a 45year old male presented with a history of dyspnoea and cough since 8months. On evaluation, a left sided pleural thickening was present and a CT guided biopsy was taken which revealed IMT. The patient was earlier treated on ATT for 6months and was advised to continue ATT for another 3 months with Oral prednisolone $30 \mathrm{mg}$ was started for 10days and tapered with $5 \mathrm{mg}$ decremental doses over a period of 4 weeks. A long term follow up is required to know about the overall outcome and survival of the patient.
\end{abstract}

Keywords: inflammatory myofibroblastic tumor, tuberculosis
Volume 7 Issue 4 - 2017

\author{
Tanya Syiem,' Rajesh Kumar,' Murali \\ Paramanandhan, ' Surendra Beniwal, ${ }^{2}$ Ramesh \\ Purohit,' 'Vishnu Lal' \\ 'Junior resident, Department of Radiotherpy, India \\ ${ }^{2}$ Associate Professor, Department of Medical Oncology, India
}

Correspondence: Murali Paramanandhan, Junior resident, Department of Radiotherpy, India

Email josh.messil9@gmail.com

Received: October 3, 2017 | Published: October 12, 2017
Abbreviations: IMT, inflammatory myofibroblastic tumor; CT, computer tomography, ATT, anti- tubercular therapy; PTB, pulmonay tuberculosis; IHC, immunohistochemistry; PETCT, positron emission tomography with computer tomography

\section{Introduction}

Inflammatory myofibroblastic tumor (IMT) of lung is a rare benign malignancy of lung. ${ }^{1}$ It histology is varied, there is combination of stromal, myoepithelial and fibrous histiocytes and plasma cells on microscopy. The incidence is rare and accounts for about $0.04-1 \%$ of all lung tumors. ${ }^{2}$ It is most commonly seen in young adults though rare in patients above 60years of age. The commonest presentation is cough, dyspnoea etc., there could be no underlying symptoms in most cases. ${ }^{3}$ Complete surgical excision is the treatment of choice. This present case report highlights a rare case of IMT in a middle age male patient diagnosed and treated for pulmonary tuberculosis was later diagnosed as IMF.

\section{Case report}

A 45year old male patient presented with a history of dyspnoea and cough since 8 months. The patient was well built and nourished. His vitals were stable. Plain radiograph of chest, CT thorax was normal. Diagnostic bronchoscopic was done, the bronchial brushing was taken and was found to be positive for pulmonay tuberculosis (PTB). The patient was started on anti-tubercular therapy (ATT). He completed 6months of therapy and there was marginal reduction in the symptoms. A repeat CT thorax was done showing pleural thickening with hyperinflated left lung and bronchiectatic changes multiple patchy areas of consolidation in bilateral lungs. A CT guided biopsy was taken from the Left pleural thickening. The microscopy showed a mixed feature of spindle cells interlaced with plasma cells and lymphocytes. Immunohistochemistry (IHC) showed positivity for smooth muscle actin and vimentin and ki-67 index was $12 \%$, the ALK was negative confirming the diagnosis of IMT. PET-CT was done which also confirmed the findings as in the CT thorax, with hypermetabolic uptake in the left pleura and bilateral supraclavicular areas. The patient was continued on ATT till 9months, as well as started on pulsed dose steroid therapy starting dose at Oral prednisolone 30mg was started for 10 days and tapered with $5 \mathrm{mg}$ decremental doses over a period of 4weeks (Figure 1-3).

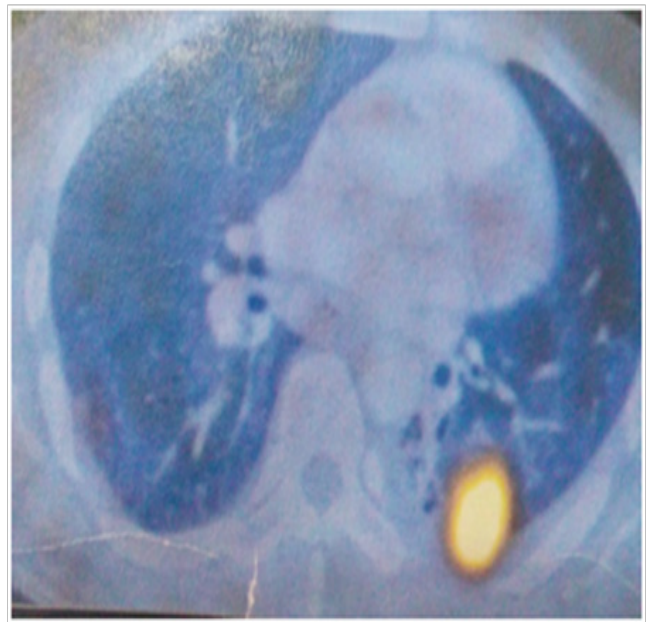

Figure I PET-CT shows hypermetabolic activity in left lung.

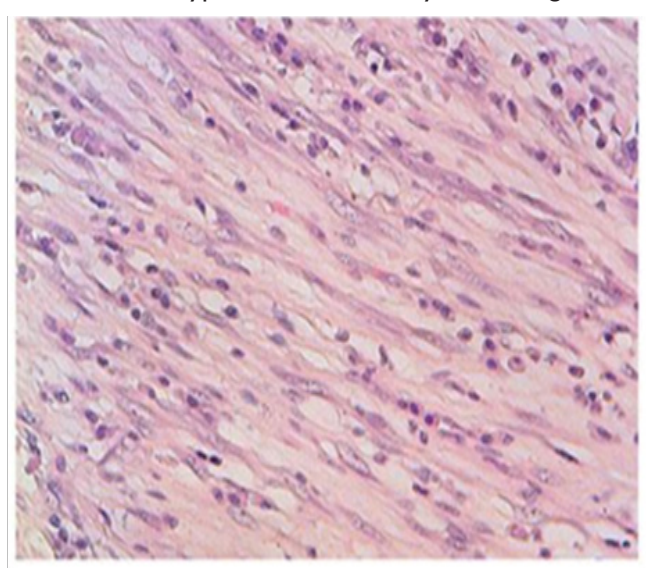

Figure 2 Proliferation of regular myofibroblasts mixed with lymphocytes and plasma cells (high magnification). 


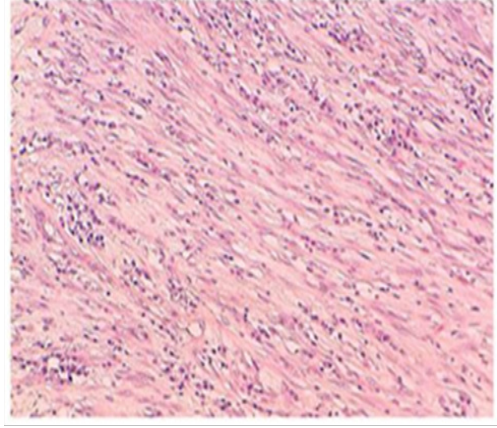

Figure 3 Spindle cells arrayed in fascicles, mixed with inflammatory cells (medium magnification).

\section{Discussion}

IFT of lung is a rare benign tumor of the lung reported in $0.04 \%$ $1 \%$ of all lung tumors. ${ }^{2}$ There is no sex predilection and has been reported most frequently seen in age of 20-50years. There have been reports of existing co-infection in some patients. The patient usually present with non-specific symptoms like cough, dysnoea, fever, chest pain, weight loss etc. There are many uncertainties about the pathogenesis of IMT. Several hypotheses have been proposed such as an auto-immune mechanism or infectious origin. Indeed, $30 \%$ of cases are closely related to recurrent respiratory infections which are caused by several microorganisms such as Mycoplasma, Nocardia, Actinomycetes, Epstein Barr and human herpes virus. ${ }^{3,4}$ In the present case, the patient is a 45year old male with history of cough and dyspnoea, with positive bronchial washings for PTB. The patient was started on ATT-DOTS therapy.

The biopsy is necessary to confirm the diagnosis, which usually shows a combination of inflammatory cells and mesenchymal cells which mainly is the plasma cells, histiocytes, lymphocytes and spindle cells Pettinato et al., ${ }^{5}$ has described IFT histologically to be a an inflammatory cells with proliferative myofibroblasts and fibroblasts, with IHC positive for vimentin and smooth muscle actin. ${ }^{6}$ The IMT can invade blood vessels but a rare occurrence, as it infiltrates the lung with the inflammatory cells, it is very difficult to differentiate as the normal lung architecture gets distorted. The imaging studies done at baseline was normal, but a repeat $\mathrm{CT}$ thorax was done showing pleural thickening with hyper inflated left lung and bronchiectatic changes multiple patchy areas of consolidation in bilateral lungs. PET-CT confirmed the findings with hyper-metabolic uptake in the bilateral supraclavicular areas. In our case the biopsy of the pleural thickening showed mixed areas of spindle cells interlaced with plasma cells and lymphocytes. IHC positive for smooth muscle actin, vimentin and ALK negative, confirming IMT.

The differential diagnosis for IMT are malignant fibrous histiocytoma, malignant lymphoma, sclerosing hemangioma, lymphoid hyperplasia, pseudolymphoma, nodular chronic pneumonitis, sarcomatoid carcinoma of the lung, sarcoma, plasmacytoma. The controversy continues to exist if IMT is a reactive or a neoplasm. These lesions can be differentiated by careful attention to cellular atypia, necrosis, mitotic activity, immunoreactivity, or clonality., ${ }^{2,7}$ Recent findings is suggestive of neoplasm because of the clonal chromosomal abnormalities. The chromosomal rearrangements involving the ALK receptor tyrosine-kinase locus region (chromosome band $2 \mathrm{p} 23$ ), or DNA aneuploidy in IMT., ${ }^{910}$ In our case this chromosomal study was not done. There for taking into consideration of the above features IFT can be considered as a low grade tumor or a benign malignancy.
The chances of distant metastasis are very rare. In our case the proliferative Ki-67 done showed 12\% which also confirmed the low malignant potential.

The treatment of choice is surgical excision ${ }^{2}$ preferably the wide local excision. In case of the lung, it should be a lobectomy or a segmentectomy. It becomes both a curative and a diagnostic procedure. Although other forms of treatment like chemotherapy, radiotherapy and steroids have been tried with variable success. ${ }^{11}$ There have been reports of wait and watch approach as there is chances for spontaneous regression once the associated inflammatory pathology is treated. In our case, the patient was initially diagnosed as PTB and started on ATT, and IMT was diagnosed as there was no drastic symptomatic improvement after the 6months. Following the diagnosis of IMT, the ATT was continued for 3 months that is in total for 9months. Also, the patient was started on steroid therapy. Oral prednisolone $30 \mathrm{mg}$ was started for 10days and tapered with $5 \mathrm{mg}$ decremental doses over a period of 4 weeks. The patient did not undergo resection as the patient was already a know case of PTB and imaging showed multiple miliary areas in bilateral lungs.

\section{Conclusion}

IMT of lung is a rare, benign malignancy. The diagnostic dilemma in the arriving at the diagnosis poses a challenge. It requires enough histological specimens to confirm the diagnosis. The best diagnostic and therapeutic measures are the surgical resection. The authors would like to conclude that a long term follow up is required to know about the overall outcome and survival of the patient.

\section{Acknowledgements}

None.

\section{Conflict of interest}

The author declares no conflict of interest.

\section{References}

1. Almadi A, Rami M, Khattala K, et al. Pseudotumeur inflammatoire pulmonaire chez un enfant. J Pediatr Puericulture. 2011;24:69-71.

2. Cerfolio RJ, Allen MS, Nascimento AG, et al. Inflammatory pseudotumors of the lung. Ann Thorac Surg. 1999;67(4):933-936.

3. Pinillaa I, Herreroa Y, Torresa MI, et al. Tumor inflamatorio miofibroblástico pulmonary. Radiologa. 2007;49:53-55.

4. Ochs K, Hoksch B, Frey U, et al. Inflammatory myofibroblastic tumour of the lung in a five-year-old girl. Interact Cardiovasc Thorac Surg 2010;10(5):805-806.

5. Ishida T, Oka T, Nishino T, et al. Inflammatory pseudotumor of the lung in adults: radiographic and clinicopathological analysis. Ann Thorac Surg. 1989;48(1):90-95.

6. Pettinato G, Manivel JC, De Rose N, et al. Inflammatory myofibroblastic tumor (plasma cell granuloma): clinicopathologic study of 20 cases with immunohistochemical and ultrastructural observations. Am J Clin Pathol. 1990;94(5):538-546.

7. Mandelbaum I, Brashear RE, Hull MT. Surgical treatment and course of pulmonary pseudotumor (plasma cell granuloma). J Thorac Cardiovasc Surg. 1981;82(1):77-82.

8. Matsubara O, Mark EJ, Ritter JH. Pseudoneoplastic lesions of the lungs, pleural surfaces, and mediastinum. In: Wick MR, et al editors. Pathology of pseudoneoplastic lesions. USA; 1997. p. 100-109. 
9. Lawrence B, Perez-Atayde A, Hibbard MK, et al. TPM3-ALK and TPM4-ALK oncogenes in inflammatory myofibroblastic tumors. Am J Pathol. 2000;157(2):377-384.

10. Biselli R, Ferlini C, Fattorossi A, et al. Inflammatory myofibroblastic tumor (inflammatory pseudotumor): DNA flow cytometric analysis of nine pediatric cases. Cancer. 1996;77:778-784.
11. Imperato JP, Folkman J, Sagerman RH, et al. Treatment of plasma cell granuloma of the lung with radiation therapy: a report two cases and a review of the literature. Cancer. 1986;57(11):2127-2129. 